\title{
Latent Space Metric Learning For Sidescan Sonar Place Recognition
}

\author{
Max Larsson ${ }^{1}$, Nils Bore $^{1}$, John Folkesson ${ }^{1}$
}

\begin{abstract}
Place recognition is an essential part of a Simultaneous Localization and Mapping, SLAM, framework. By recognizing that the AUV has returned to a previously visited place, constraints on the trajectory can be imposed leading to a reduction in navigation drift during a mission. The aim here is to recognize previously visited places solely from sidescan data using a neural network. Image patches are fed to a ResNet type architecture to extract place descriptors. Triplet loss metric learning is used for training. Results on actual sidescan data are shown.
\end{abstract}

\section{INTRODUCTION}

Place recognition in sidescan sonar images is important for AUV navigation using Simultaneous Localization and Mapping, SLAM. Recognizing distinctive features on the seabed could be used to trigger a so called loop closure. These detected loops create the constraints on the AUV trajectory that are needed for SLAM to be effective in reducing drift in navigation [1], [2].

A SLAM framework contains several parts: the Bayesian optimization engine forms an a posteriori estimate of the map and trajectory given both the motion and environmental measurements; the data association allows proper modelling of measurements; and the loop detection tells the data association when and where to start looking for measurements to model. That is, given a sequence of measurements, it is able to associate measurements taken at different times by identifying them as coming from the same area. It is this loop detection that we are addressing here.

Due to its thin, elongated shape, sidescan sonar is wellsuited for mounting on smaller AUVs. When comparing to multibeam sonars, the resolution of its measurements and cost considerations also play to its advantage. However, in choosing sidescan, one sacrifices the multibeam's ability to directly determine the seafloor geometry. Taken together, sidescan is often a good sensing option for smaller AUVs, and might be the only option if its shape is elongated. For SLAM, both sidescan and multibeam have the disadvantage of only seeing the terrain once, unless the AUV's path takes it back to a previously visited area. Thus the sidescan will not help reducing the continuous drift in dead reckoning the way that cameras can do for robots on land. This is done in camera images by matching features in the images in adjacent frames in the sequence.

Sidescan can be used, however, to reduce the drift when there is overlap in the ensonified portion of the seabed upon

\footnotetext{
*This work was supported by Stiftelsen för Strategisk Forskning (SSF) through the Swedish Maritime Robotics Centre (SMaRC) (IRC15-0046).

${ }^{1}$ Robotics, Perception and Learning Division at KTH Stockholm, Sweden \{maxlars, nbore, johnf\}@kth. se
}

return to an area. This might happen for example during a survey of parallel lines when the sonar can see further than half the distance between the lines. If we identify the same physical features in measurements along both lines, the drift of the second line with respect to the first can be removed, up to an error in the formed constraints. As there might be many such constraints along the line, the SLAM optimization could in principle reduce the error significantly.

In order to form a constraint, a transformation between two sidescan patches needs to be computed based on details in the two images. In this current work, we instead focus on loop closure detection through place recognition. In a full SLAM system, the place recognition method will then trigger such a constraint computation, effectively telling the system to add a constraint between two patches of sidescan. As such a system does not provide a geometrical model of the constraint, its main use is to identify places after very long loops with much drift. It may also be used for approximate localization to a prior 'map' consisting of a database of previous georeferenced images of the environment.

The main contributions here are to show that it is possible to recognize places using sidescan images in some environments and to propose a data driven method for doing so. We show that on real data the method can identify places previously seen with relatively high accuracy. This would be sufficient to be used in a SLAM framework to be followed by a fine (pixel level) matching step that could both compute the transformation between the AUV positions and identify false positive matches. It could also be used in a similar way for localization to a previously surveyed part of the seafloor, if the environment has not changed significantly since the prior map was made.

\section{RELATED WORK}

Place recognition in camera images first used so called hand crafted features such as SIFT [3], and SURF [4]. More recently, deep neural networks, so called data driven methods [5], [6], [7] are being applied. The data driven methods have been more successful but require training data which is difficult to collect, particularly underwater. Furthermore, camera images on land are more varied in types of features, objects, buildings, sign posts, etc., than a normal underwater scene which mainly contains rocks and sediment. In addition, underwater cameras are not very useful for localization over large areas as they have limited range. Since the range of sidescan often extends to an order of hundreds of meters, it does not face the same challenges as underwater cameras. It is therefore possible that sidescan images may be used 
in ways analogous to how camera images have traditionally been used in land-based localization.

In [1] sidescan sonar was used in a SLAM framework. The SLAM engine was iSAM and the sidescan image feature detection, loop detection, and data association were done manually. They point out that sidescan images are more difficult than camera images to use for these tasks. The main difficulties include a strong dependence of appearance on viewing angle, a lack of variability in the feature types and shadow being the most detectable part of features, but shadows do not match the position of the feature.

Most approaches to place recognition start by building a description of the image. An algorithm then searches a database of past images to find one with a similar description. A classic method builds up a dictionary of 'words' based on clustering in a local feature descriptor space. A typical image contains many such feature 'words' and thus a histogram over 'word' counts in the image can be a description of the image [6], [8], [9], [10].

Neural networks learn to encode the image directly into a descriptor space, aka the latent space. The encoder can be trained to optimize the latent space for a specific task such as place recognition. Due to the large datasets available for image classification, there are well developed network architectures for that task. These can then be used for other tasks with some more limited training for the specific task. For example the ResNet image recognition [11] network, which we use here (but not pretrained), can be used for other tasks in this way. An example of using a pretrained network for place recognition in camera images is [12]. In that work they compare using hand crafted features to the features generated by various layers of a pretrained network. They conclude that the CNN generated features were more robust to lighting changes while performing similarly otherwise.

In [13] place recognition in forward looking sonar is demonstrated using a similar metric learning method to ours. Forward looking sonar has some similarity to sidescan sonar, but the images typically cover a much smaller region of the sea floor. They use PoseNet [14] as the structure for the neural network encoder.

\section{Metric LeARning Method}

We will use an 18 layer ResNet [11] to learn a mapping, $Y=f(X)$, of sidescan image, $X$, to a latent representation, $Y$. The encoder trained on camera images will produce some latent representation of a sidescan image but this will not be very good for the task of place recognition in sidescan images. For that reason we initialize and train the network from scratch to solve our specific problem. To produce a good latent representation we need to force overlapping images to appear close in the latent space while non-overlapping ones are well separated. This is known as metric learning.

The triplet loss approach, [15], [16], to metric learning forms a loss specifically tailored to separate the latent representations in this way. A triplet consists of three samples, anchor, positive and negative. The anchor sample is a randomly selected sample. The positive sample is taken from the same area of the seafloor as the anchor sample and the negative sample is taken from a different area.

In our case, a positive sample is a sample that has an overlap value $O>=\tau$ with the anchor sample and a negative sample is a sample where $O=0$ with the anchor sample, where $\tau$ is defined to be the overlap threshold. All samples with overlap values between $0<O<\tau$ are discarded and are neither regarded as positive, nor negative. For an example of a triplet, see Fig. 1. Those images are small patches cut from the much larger sidescan image.

The loss function for a single triplet is:

$$
\mathscr{L}(A, P, N)=\max \left(\|f(A)-f(P)\|_{2}-\|f(A)-f(N)\|_{2}+\alpha, 0\right) .
$$

where $f(A), f(P)$ and $f(N)$ are the feature vectors for the anchor, positive, and negative sample respectively, $\alpha$ is a positive constant, and $\|\cdot\|_{2}$ is the L2-distance. All feature vectors, $f(\cdot)$ are normalized to have the length 1 .

Naturally there are many more negative samples than positive samples for each anchor sample. A hard negative sample is defined as $H=\operatorname{argmin}_{N}\|f(A)-f(N)\|_{2}$, where $N$ is an arbitrary negative sample. A semi-hard negative sample is where $\alpha>\mathscr{L}(A, P, N)>0$. Semi-hard samples are further from the anchor than the positive sample, but not by more than $\alpha$, while the hard sample is the closest negative sample to the anchor.

All possible triplets could of course be used, but that would lead to slow convergence, due to the vast amount of triplets where the loss function would be 0 . Given the low number of positive samples, we decided to use them all. If sample $i$ first was selected as an anchor sample and sample $j$ was a selected positive sample, then if during the same epoch sample $j$ was selected as anchor sample, sample $i$ was not allowed to be a positive sample to sample $j$. The same goes for any given negative sample. The network was trained on mini-batches of size $n=200$ samples, where all possible positive samples, within the mini-batch, were used and the corresponding negative samples were selected so that they were semi-hard. If no semi-hard negative sample existed, we instead used the hard negative sample for that triplet.

The proposed network architecture is an 18 layer ResNet [11] with one channel in the first layer instead of the typical three, since we do not work with RGB images. The last layer, the feature embedding, is a fully connected $128 \times 1$ vector. For back propagation stochastic gradient descent was used.

Before training the network, a randomized set of validation triplets were created. First, the network was trained for 100 epochs with a learning rate of 0.05 . The model parameters, in the epoch with the highest percentage of correctly classified validation triplets, were saved. Using the saved model parameters, the network was trained for another 50 epochs with a lower learning rate of 0.005 and the best set of model parameters were once again saved. The momentum parameter was set to 0.3 for all epochs. 

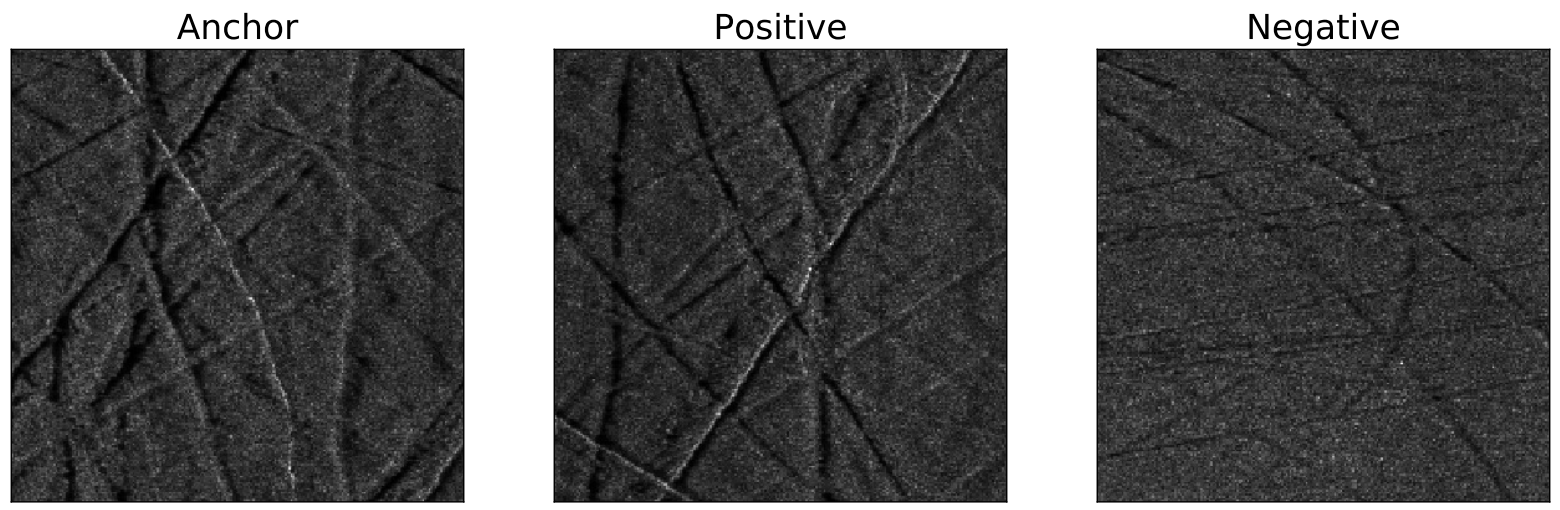

Fig. 1: Example of a triplet with overlap threshold, $\tau=0.6$. The scoring pattern is caused by trawling.

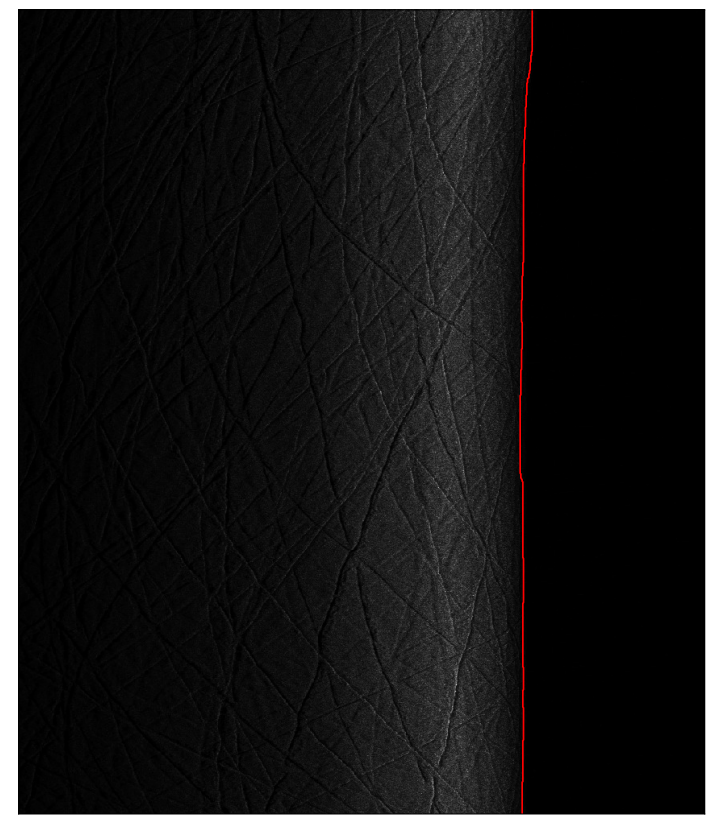

(a) The port side of the original waterfall image.

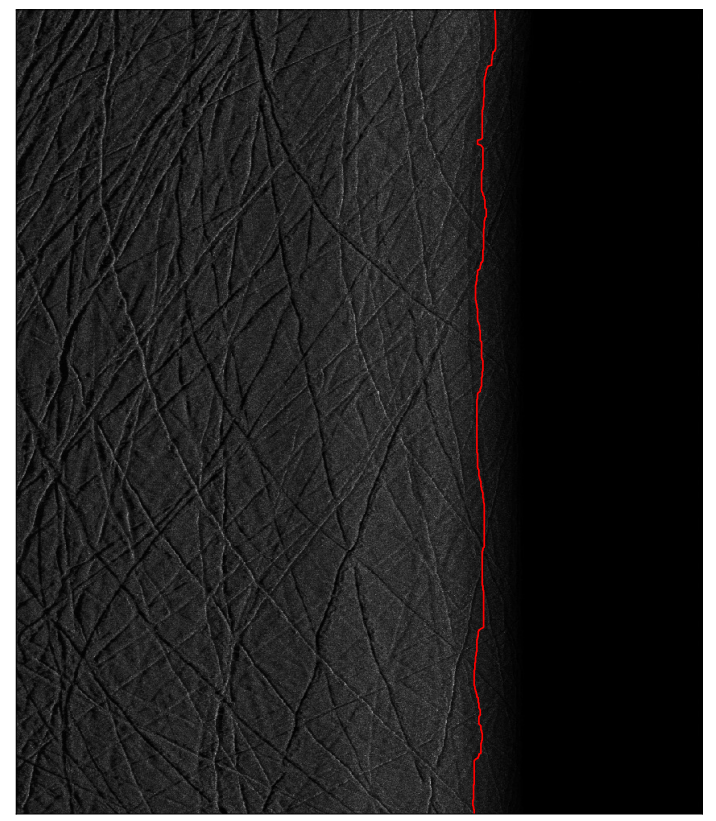

(b) The port side of the normalized waterfall image.

Fig. 2: Waterfall images with detected nadir, using the Canny edge detector.

\section{Sidescan Data SETS}

\section{A. Data Collection and Preprocessing}

The sonar data was collected on the west coast of Sweden with Gothenburg University's HUGIN AUV. The sidescan sonar was an Edgetech 2200-M and the multibeam sonar (MBES) was a Kongsberg EM2040. The first survey ran parallel lines over the area then repeated with the direction rotated by 90 degrees. The second survey covered the same area with lines in one direction only but much closer together (10m) which gave more overlap between more lines. The observed area consists of relatively flat sediment, but does contain scoring patterns from trawling. The hypothesis was that these lines made the region salient enough to perform feature detection with a CNN on the sidescan sonar data.

We first normalize the image to reduce the effect of incidence angle to the relatively flat bottom, see Fig 2 for an example. This normalization assumes a flat horizontal bottom and a $\cos ^{2} \phi$ dependence of intensity to incidence angle. The range of the nadir, detected with a canny edge detector, is used to set the altitude of the sonar above this flat bottom. The normalized waterfall images have sections cropped from the region well beyond the nadir where the shallower grazing angle gives us better resolution. This outer region on both sides of the nadir will be referred to as the working region. All samples in our final dataset will be taken from the working region, see Fig. 3.

\section{B. Generating Samples}

The normalized waterfall images without downsampling are each 19912 pixels wide. During the creation of the waterfall images, to reduce noise, we downsample them to have a width of 2048 pixels. From the downsampled, normalized waterfall images we crop out samples of size $224 \times 224$ pixels using the following cropping scheme: Starting from 


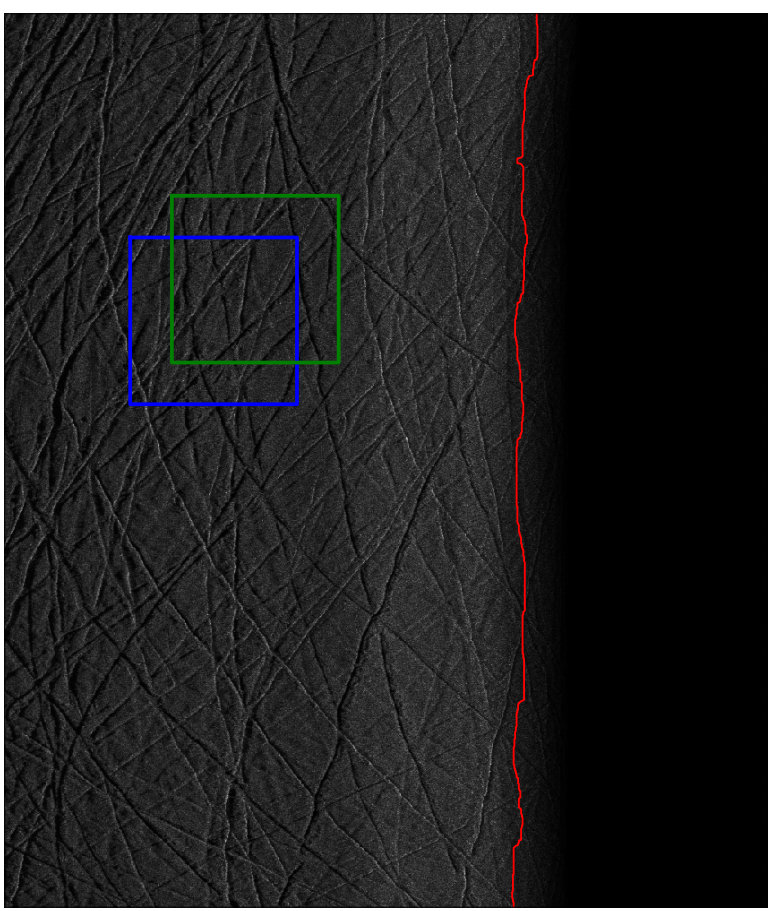

(a) Two data samples cropped from the port side.
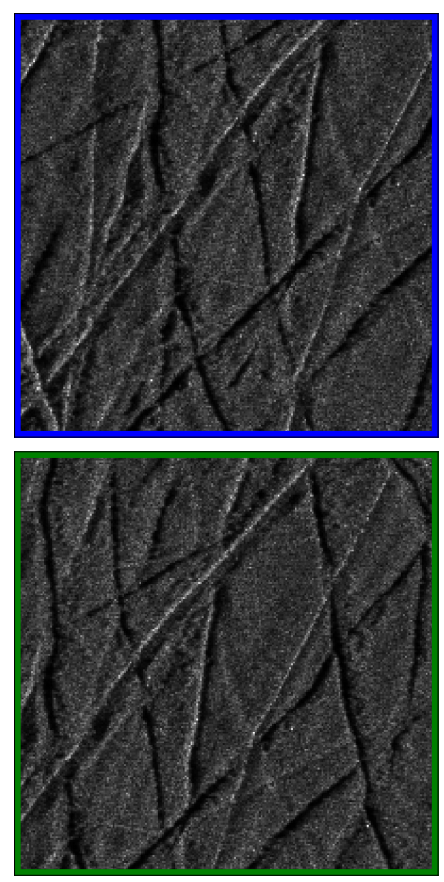

(b) Closer look at the same samples.

Fig. 3: Highlighting two $224 \times 224$ data samples used to train the network. The training set used samples such as this with overlap within the same line as positive samples, but the testing set did not.

the bottom outer corner on the port side, where we have the furthest returning intensities of the first time steps, we crop out a sample of size $224 \times 224$ pixels. Thereafter we slide this $224 \times 224$ window horizontally with a step size of 56 pixels and continuously add the corresponding samples to our data set. When it is impossible to go further horizontally without crossing the nadir line, we move up 56 pixels vertically, and once again start going horizontally towards the nadir from the outer edge. This process is repeated for all waterfall images, for all survey lines. The samples are then shuffled and divided into a training, validation and test set. The final number of samples are 5060 training, 2064 validation, and 1164 testing samples. The training samples were used to form 45590 triplets. From the validation samples we got 7520 triplets. An overlap threshold of .2 was used for both the training and validation triplets. The training and validation sets come from the first survey and the test set from the second survey.

The MBES data was used to generate a 3D mesh of the seabed, allowing us to geo-reference each of our cropped sidescan patches. Each pixel of the samples are thus represented by a 3D spatial coordinate on the generated mesh.

We define $A(s)$, the area on the mesh, for a given input sample $s$. To quantify the degree to which two samples are from the same location, each pair of samples gets assigned a number between $0-1$, corresponding to how much the samples are overlapping on the bathymetry mesh. 0 means no overlap, and 1 means that the two samples cover the exact same area. In general the overlap between two samples is defined as:

$$
O=\frac{A\left(s_{1}\right) \bigcap A\left(s_{2}\right)}{A\left(s_{1}\right) \bigcup A\left(s_{2}\right)},
$$

where $O$ is the overlap, $s_{1}$ and $s_{2}$ are two different samples, and $A(\cdot)$ is the previously mentioned area-function.

\section{EXPERIMENTS AND RESULTS}

Fig. (4) shows an example of the progress during the training of the accuracy on the validation set for the .2 overlap threshold. The training and validation sets were taken from the first survey which did not have as many positive samples as the second survey. For that reason we included as positive samples cropped patches from the same line that overlapped. These are of course much easier to recognize than the same area seen in different lines. The easier samples give us more triplets to train with. Training should go faster using this extra data. The test set did not include positive matches in the same line in the evaluations. We evaluated the trained networks in two ways: accuracy in classifying the positive sample as closer than the negative one on separate test sets with different overlap thresholds, Table (I); and the retrieval accuracy on the whole database of test samples, Fig. 5. The retrieval accuracy is evaluated by ranking all samples from the second dataset by their similarity to the current query sample. We then show the percentage of truly overlapping samples as a function of the position on the ranked list which we called retrieval index. As each query sample only has a limited number of positive matches in the database the plots of accuracy vs. retrieval index can only 


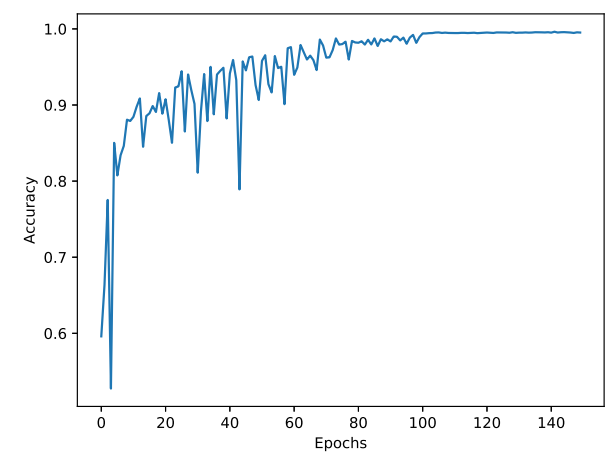

Fig. 4: The learning progress of validation accuracy, overlap threshold $\tau=0.2$. Accuracy is defined as number of correctly classified triplets, divided by total number of triplets.

go as far as we have meaningful statistics. We truncated the curves at retrieval index $\mathrm{n}$ when the number of query samples with $\mathrm{n}$ positive matches dropped below 500 .

It is interesting to see how the physical positions of the patches along the seafloor get mapped to the latent space and vice versa. In Fig. (6) we can see in the top right image that the latent space projection separates the patches into clusters. Within each cluster the colors change continuously, keeping red together for example. The bottom right image shows that the direction of the lines that the patch was taken from has been encoded into the latent space. This separation means that the network may find it difficult to match the same place seen from 90 degree different angles.

\section{Discussion}

The accuracy result, Table (I), with .2 overlap threshold is better than random guessing $(50 \%)$ and for higher overlap the method gives fair results. These results are between individual patches. For a place recognition system the idea would be to extract many patches from the current waterfall and then try to find a consistent set of matches to the database. So low accuracy could well be sufficient for robust place recognition. The retrieval task results, Fig. 5, shows that the closest patches will often contain some positive matches, again indicating that this might be practical when coupled with logic to eliminate the false positives.

Further work is needed to investigate the accuracy on patches seen from perpendicular directions.

\section{CONCLUSIONS}

General conclusions can not be drawn without more data from other environments, but we have shown that it is possible to recognize patches of seafloor in sidescan images if the right sort of bottom features exist. The image retrieval result indicates that a localization method could work using sidescan images.

\section{ACKNOWLEDGEMENT}

This work was supported by Stiftelsen for StrategiskForskning (SSF) through the Swedish Maritime Robotics

\begin{tabular}{|l|l|l|l|}
\hline Overlap Threshold $(\tau)$ & $\mathbf{0 . 2}$ & $\mathbf{0 . 4}$ & $\mathbf{0 . 6}$ \\
\hline Acccuracy & 0.6968 & 0.7350 & 0.7600 \\
\hline Number of triplets & 49745 & 17491 & 4905 \\
\hline
\end{tabular}

TABLE I: Accuracy on the test sets of triplets for different overlap thresholds. Accuracy is defined as number of correctly classified triplets divided by total number of triplets in the set.

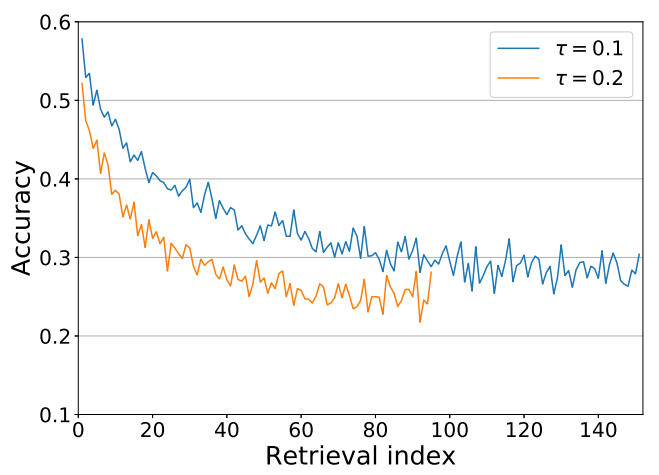

Fig. 5: The accuracy at retrieval index of $\mathrm{k}$ means the $k^{\text {th }}$ ranked sample patch, when the entire database is compared using cosine similarity, has a larger overlap than $\tau$ that often.

Centre (SMaRC) (IRC15-0046). We also thank Professor Anna Wåhlin for help in collecting the data.

\section{REFERENCES}

[1] M. F. Fallon, M. Kaess, H. Johannsson, and J. J. Leonard, "Efficient auv navigation fusing acoustic ranging and side-scan sonar," in Robotics and Automation (ICRA), Shanghai, China. 2011 IEEE International Conference on, pp. 2398-2405, IEEE, 2011.

[2] M. Fallon, J. Folkesson, H. McCelland, and J. Leonard, "Relocating underwater targets autonomously using sonar-based slam," IEEE Journal of Ocean Engineering, pp. 1-14, 2013.

[3] D. G. Lowe, "Distinctive image features from scale-invariant keypoints," International journal of computer vision, vol. 60, no. 2, pp. 91-110, 2004.

[4] H. Bay, T. Tuytelaars, and L. Van Gool, "Surf: Speeded up robust features," in Computer Vision - ECCV 2006 (A. Leonardis, H. Bischof, and A. Pinz, eds.), (Berlin, Heidelberg), pp. 404-417, Springer Berlin Heidelberg, 2006.

[5] K. M. Yi, E. Trulls, V. Lepetit, and P. Fua, "Lift: Learned invariant feature transform," in Computer Vision - ECCV 2016 (B. Leibe, J. Matas, N. Sebe, and M. Welling, eds.), (Cham), pp. 467-483, Springer International Publishing, 2016.

[6] D. Galvez-López and J. D. Tardos, "Bags of binary words for fast place recognition in image sequences," IEEE Transactions on Robotics, vol. 28, no. 5, pp. 1188-1197, 2012.

[7] N. Suenderhauf, S. Shirazi, A. Jacobson, F. Dayoub, E. Pepperell, B. Upcroft, and M. Milford, "Place recognition with convnet landmarks: Viewpoint-robust, condition-robust, training-free," in Robotics: Science and Systems XI (D. Hsu, ed.), pp. 1-10, http://www.roboticsproceedings.org/: Robotics: Science and Systems Conference, 2015.

[8] Sivic and Zisserman, "Video google: a text retrieval approach to object matching in videos," in Proceedings Ninth IEEE International Conference on Computer Vision, pp. 1470-1477 vol.2, 2003.

[9] G. Csurka, C. Dance, L. Fan, J. Willamowski, and C. Bray, "Visual categorization with bags of keypoints," in Workshop on statistical learning in computer vision, ECCV, vol. 1, pp. 1-2, Prague, 2004.

[10] M. Cummins and P. Newman, "Appearance-only slam at large scale with fab-map 2.0," The International Journal of Robotics Research, vol. 30, no. 9, pp. 1100-1123, 2011. 


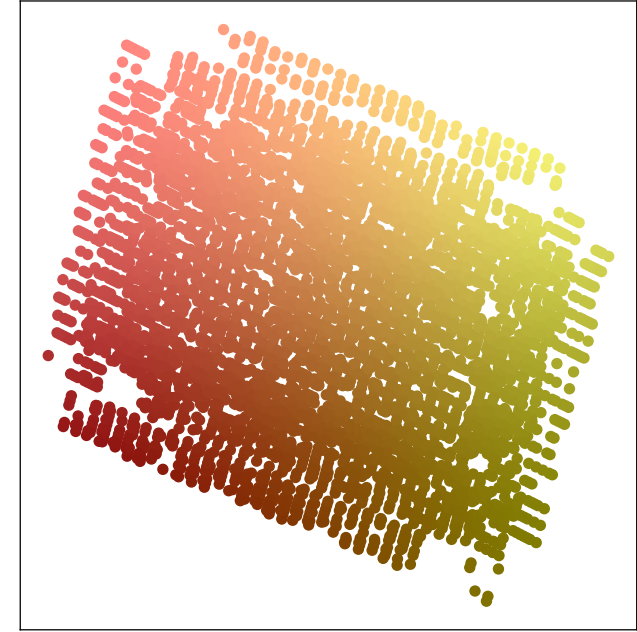

Latent Space

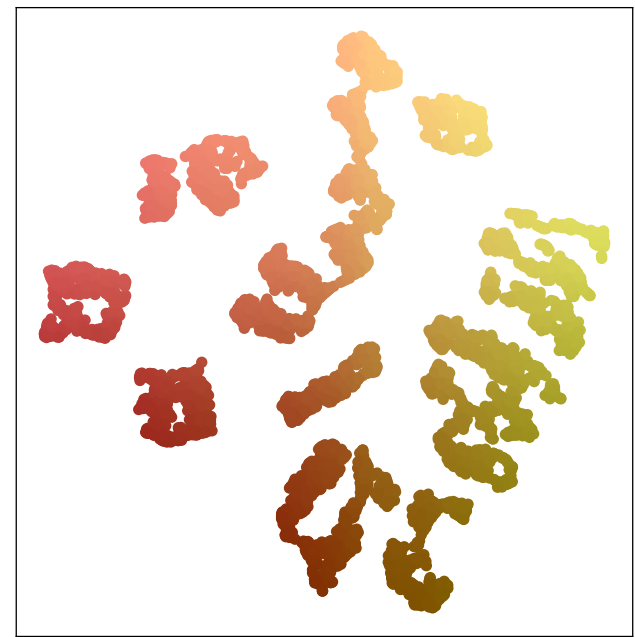

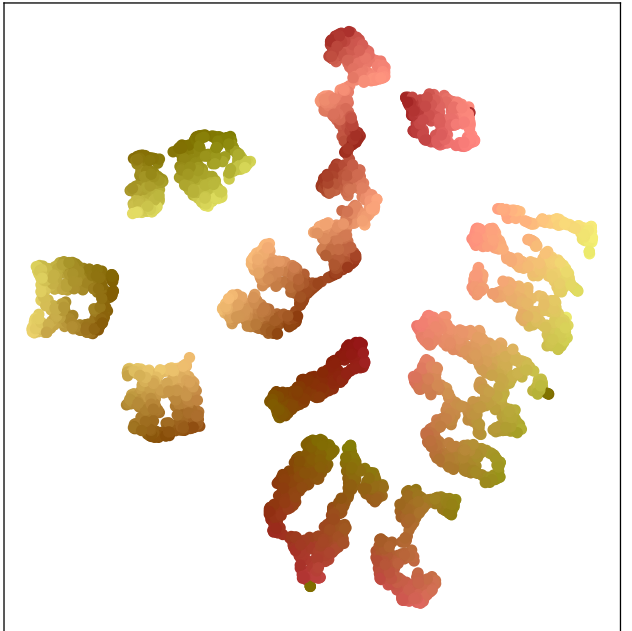

Positions

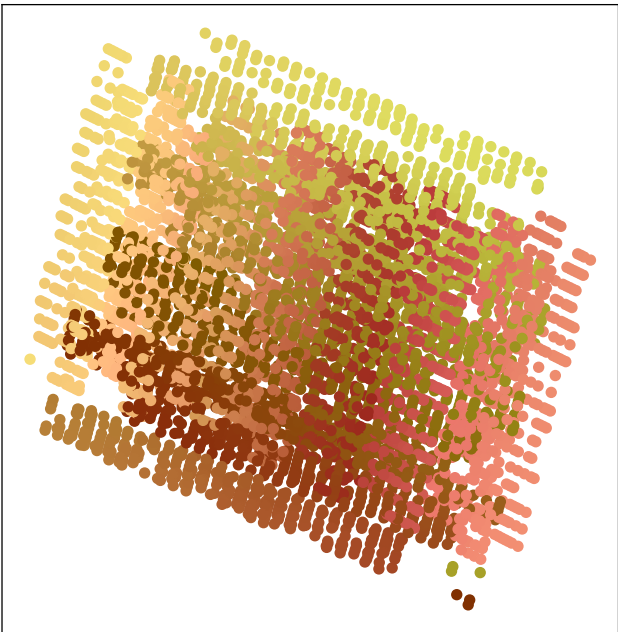

Fig. 6: Each disk represents a training data sample. The left column has the color map with light to dark top to bottom and red to green left to right. The right column shows the same samples mapped to the other space. The position space is $\mathrm{x}$ and $\mathrm{y}$ coordinates along the sea floor. The latent space is a projection to $2 \mathrm{D}$ of the latent space. The mapping was done using t-SNE.

[11] K. He, X. Zhang, S. Ren, and J. Sun, "Deep residual learning for image recognition," CoRR, vol. abs/1512.03385, 2015.

[12] Y. Hou, H. Zhang, and S. Zhou, "Convolutional neural network-based image representation for visual loop closure detection," in 2015 IEEE international conference on information and automation, pp. 22382245, IEEE, 2015.

[13] P. O. C. S. Ribeiro, M. M. dos Santos, P. L. J. Drews, S. S. C. Botelho, L. M. Longaray, G. G. Giacomo, and M. R. Pias, "Underwater place recognition in unknown environments with triplet based acoustic image retrieval," in 2018 17th IEEE International Conference on Machine Learning and Applications (ICMLA), pp. 524-529, 2018.

[14] A. Kendall, M. Grimes, and R. Cipolla, "Posenet: A convolutional network for real-time 6-dof camera relocalization," in Proceedings of the IEEE international conference on computer vision, pp. 2938-2946, 2015.

[15] V. Kumar B G, G. Carneiro, and I. Reid, "Learning local image descriptors with deep siamese and triplet convolutional networks by minimising global loss functions," in The IEEE Conference on Computer Vision and Pattern Recognition (CVPR), June 2016.

[16] F. Schroff, D. Kalenichenko, and J. Philbin, "Facenet: A unified embedding for face recognition and clustering," CoRR, vol. abs/1503.03832, 2015 